\title{
Broadband Access for All: Strategies and Tactis of Wireless Traffic Sharing
}

\author{
Jinadu Olayinka T. \\ Department of Computer Science, \\ Rufus Giwa Polytechnic, Owo, \\ Ondo State, Nigeria
}

\author{
Owa Victor K.. \\ Department of Computer Science, \\ Rufus Giwa Polytechnic, Owo, \\ Ondo State, Nigeria
}

\begin{abstract}
Network engineers have designed an array of protocols that enabled shared access in various wired and wireless contexts at different layers of the protocol stack [1]. One approach to managing unlicensed spectrum is to rely on a technical protocol to allocate and manage shared access Lehr [2]. This paper addressed the benefits of unlicensed wireless traffic within licensed traffic (anticipated as cognitive router-based networking). A focus on shared access to non-exclusive use of the spectrum with an holistic view of technical and institutional features is suggested for effective management of 'spectrum commons'. Using the adapted cognitive radio architectural model and its associated multi-hop ad-hoc networking strategies to implement the 'spectrum-common', mobility is enhanced with each node acting as a router and packet forwarder. We formulate management frameworks that can integrate well with liquid protocols for mobile nodes. Also, these frameworks incorporate new strategies of intelligently adapting the nodes to dynamically participate in setting bandwidth capacity stochastically. The projected use of dynamic bandwidth shaping algorithm for the cognitive radio-based network (CRN) when implemented will make broadband access more economical to users and the spectrum used effectively.
\end{abstract}

Keywords-ad-hoc; spectrum commons; etiquettes; software defined radio (SDR); MIMO

\section{INTRODUCTION}

The significant progress in wireless technology and the growth of wireless services has provided the principal impetus for reforming spectrum management and hence the transition toward increased reliance on market forces. While many wireless technologies contribute to both the viability and desirability for managing spectrum via unlicensed (smart wireless system technology, including software or cognitive radios, smart antennas and multiple input multiple output MIMO system) platforms, the benefits of unlicensed wireless are best anticipated in the context of ad-hoc networks [3].

The ad-hoc networks are mobile, dynamic wireless networks that require no fixed infrastructures [4]. As the continuous end-to-end connectivity between its mobile nodes is not guaranteed, [5] pointed out that the ability to self-form and self-mange remains a major challenge. Due to this partial and intermittently connected wireless frameworks, the mobile adhoc network (MANET) hosts induce link disruptions, which may result to degradable service disruptions except assisted by derived technologies including intelligent etiquettes and strategized mobility management.
Today, spectrum licenses to provide mobile services offer an entry barrier that gives incumbent licensee a strategic advantage. However, with robust competition and the threat of increased allocation for competing wireless technologies on one hand and the prospect of having to pay for additional spectrum to support new ( $3 \mathrm{G}$ wireless broadband) services on the other, the mobile operators are more inclined to share spectrum [1] and [2].

\section{A. Motivation}

As policy-makers are committing to a dual regime of flexible licensed and unlicensed spectrum to provide for the evolution from the centralized approaches to more decentralized management regimes, the elements of a protocol for managing the spectrum commons must be defined. These new protocols are required both at the level of running codes (as protocols and standards) and at the level of institutional frameworks.

Also, as wireless traffic become more like Internet traffic with heterogeneous, bursty or fat-tailed, long-hold time for connectivity but variable link status due to ad-hoc networking, there is need to deploy now strategies to manage wireless resources [6]. The proposed rules was examined for expected performance and support for ad-hoc communications with reduced overheads but increased quality of service (QoS) in [1].

\section{B. Objectives}

The objectives of this research are to:

- define suitable framework and CR-based infrastructure for a spectrum commons;

- incorporate learning strategies to make the defined protocols liquid and

- suggest approaches of incorporating defined etiquettes into existing management protocols to achieve sharing goals.

\section{REVIEW OF RELATED WORKS}

\section{A. Regulatory Models}

Reference [2] identified three models of spectrum management to include commands and control ( $\mathrm{C} \& \mathrm{C}$ ), property rights (as licensed) and open access (as unlicensed) users. 
As discussed, C\&C is a scheme whereby the government acts as the regulatory agency such as the Federal Communication Commission (FCC) in the US or Ofcom in the UK. Here, the government controls the choice of technology, spectrum uses and users. According to [7], this system is vulnerable to influence costs. As the government regulators lack the expertise to make informed decisions, the regulation is often slow and expensive, and therefore it is criticized as nonmarket-based approach [8].

In contrast, the licensed (property rights or exclusiveflexible use) and unlicensed (open access or 'commons') models are approaches stylized as market-based because the decision making power is decentralized to the market. In these schemes, the service providers, equipment makers and endusers interact and compete in the market place to determine spectrum usage.

Reference [7] further explained that even as the licensed scheme confers a property right on the licensee to use the spectrum exclusively, there are rules, which limits its tradability and licenses are subject to term limits. In the same vein, the assumption of relative spectrum abundance is provisioned by the unlicensed scheme. Reference [9] also corroborated the unlicensed model as an open access scheme operated as a 'commons' approach, where the right to access or use the spectrum is shared among users. though, under the licensed approach, an exclusive use license assigned may be traded in secondary markets, and licensees only have flexibility in the choice of technology and services offered. In addition, licensees are just allowed to trade the usage rights conferred by the license.

As the commons approach provide the right to access the spectrum in a shared manner (among the users) subject to protocols, the decision-making authority is decentralized to those who share access to the commons, and as the protocol embodies the mechanism for managing the spectrum, the decision -making is governed by the protocol put in place. Moreover, much flexibility is offered in commons even as the choice of the protocol may be made by the government or by the market via industry standardization unlike in license regime, where decision-making resides only with the central planner (government).

However, the 'commons' approach does not suggest that the spectrum will be free but it is open access to only those who conform to the unlicensed protocol. Furthermore, the unlicensed does not mean unregulated as costs incurred will be borne by users, either directly through access payments or indirectly through taxes, protocol implementation cost or congestion-related quality of service effects. These costs include costs of setting up and operating the management procedures such as processing costs to implement sharing protocol, its enforcement and control congestion. This is also borne in license.

Several additional distinctions between the licensed and commons are noted in [2]. They are both 'shared' in the sense that multiple devices and end-users simultaneously access and use the spectrum. For example, mobile operators share spectrum over multiple users, and competition among operators offers competition across technologies and markets. Also, they are both market- based and as these models offer dynamic spectrum access and movement by end-users via roaming and switching among operators, the mobile customers are secondary licenses who get to use the spectrum on the basis of rules established by the licensed operators

\section{B. Communication Standards}

The standard for modern telecommunication networks is to offer $99.999 \%$ availability. References [10], [11], [12] and [13] all discussed the role of unlicensed (commons) regimes as sure step towards providing solution to spectrum scarcity and a promoter of innovations in telecom services.

The rules for managing a spectrum commons as stipulated in [12] and [14] showed that centralized resource allocation mechanisms (ATM, token ring) provide more assurance of bounded access delays while distributed protocols (TCP, Ethernet) provide similar delays when networks are lightly loaded. Centralized approaches are less robust in dynamic state of ad-hoc networks, which characterise future wireless environments.

Similarly, VolP perfectly co-exist with FTP, email and other data traffic when the network is not congested. With TCP and IP segmentation of packets in transport, IP hop-by-hop and TCP (end-to-end) provides the special controls of allowing packets in variables length. As remarked in [2], much of the licensed spectrum (ISM band) used by Wi-Fi, Wireless LAN or Bluetooth is managed in a decentralized way analogous to the Internet and the applications are adaptive making resources isolation less strictly managed.

For these and many other standards to be effectively upheld to provide broadband access for all and BGP providing inter domain routing support, a more decentralized approach may be the only feasible way to manage resources. This also includes decoupling of spectrum frequencies from infrastructure investment and applications.

\section{DESIGN FRAMEWORK FOR 'SPECTRUM-COMMONS'}

The design of an appropriate framework for managing unlicensed spectrum is conceived to be minimally constraining but very consistent with orderly management of shared access spectrum.

Development of framework or rules structured for operating unlicensed devices to co-exist with licensed devices as primary users in dedicated unlicensed spectrum is crucial to the sharing.

\section{A. Spectrum Sharing Platform}

The environment of mixed regimes as (fig.1) provides for bulk of spectrum allocated via licensed and market-based unlicensed use. With cognitive radio network architectures and the dynamism exhibited by ad-hoc networking, the framework model is evolving, promoting innovations, and minimizing regulatory distortions. The design support marginal adjustments between licensed and unlicensed users, and within unlicensed supporting all changing protocols as need arises [7]. 


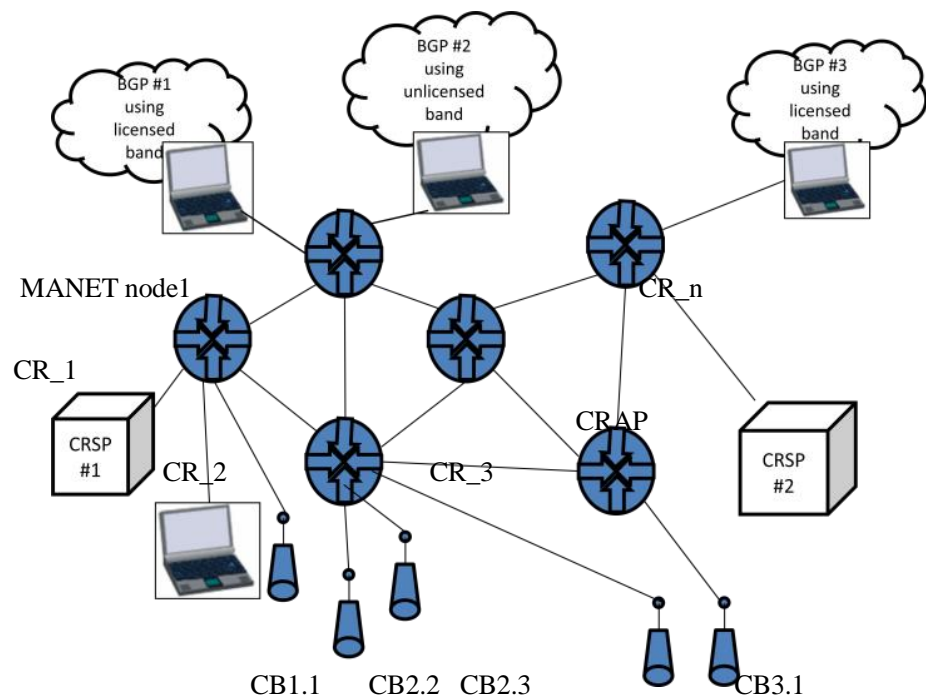

Key:

CRAP - Cognitive Radio Access Point

$C B$ - Cognitive Base

$B G P-$ Border Gateway Protocol

CRSP - Cognitive Radio Service Provision

MANET - Mobile Ad-hoc NETwork node

Fig. 1. Prototype Mesh model for CRSP (adapted from CRSN architectures)

The proto-typical design includes licenses and unlicensed bands running BGP and the radio systems made smarter. This architecture enables dynamic spectrum sharing and the framework favours distributed/decentralised management characterised with maximal 'common' benefits. Reference [9] posited set of etiquettes as rules and mechanism to instantiate a common regime. It includes 'protocol' of running code for a software radio and technical standards for guiding the protocol design for a closed common.

Fig. 1 depicted a 'closed spectrum-common' platform for licensed and qualified operators (spectrum users) to implement the management regime for spectrum usage efficiency. A collective ownership of $3 \mathrm{G}$ spectrum and its management regime is prototyped as a "closed spectrum common) in this paper.

\section{B. Design Rules}

In agreement with [15] and [16], an infrastructural framework proposed to support the traffic sharing under secured Internet routing defined by BGP is characterised with:

- technology and associated capabilities to counter communication problems such as interception, interference, eavesdropping, spoofing, jamming, data falsification etc);

- frequency agility, expanded capacity for sharing, no transmit only device spreading spectrum capability and , transition to broadband platform;

- network provisioning for bursty traffic, multimedia services and other profiles;
- heterogeneous network technology provisions 3G, Wi$\mathrm{Fi}$, Infrared, satellites roaming and seamless mobility and

- spectrum reform policies, transits to expand flexible licensing and unlicensed spectrum management regimes instituted and sustained by defined etiquettes

\section{IMPLEMENTATION OF A LIQUID PROTOCOL}

Wireless traffic control schemes for broadband services includes constant bit-rate (CBR), variable bit-rate (VBR) unspecified bit rate (UBR), guaranteed frame-rate traffic flow (GFR) and available bit-rate (ABR) service categories [17].

For liquidity, the available bit-rate (ABR) scheme is envisioned to work in the spectrum commons. ABR scheme is capable of dynamically adjusting to the varying bandwidth capacity. The bandwidth made available to an ABR connection on any link varies between minimum cell rate (MCR) and the peak cell rate (PCR).

The learning automation is a sextuple defined in [17] as

$<\mathrm{A}, \mathrm{B}, \mathrm{P}, \mathrm{T}, \mathrm{G}, \mathrm{E}>$

Where

$A=\left\{a_{l}, a_{2}, \ldots, a_{r}\right\}$ is the set of $r$ actions offered by environment

$B=(0,1)$ the input set of possible environmental responses.

$Q=$ set of possible internal states of the automation

$P=$ probability distribution over set of action $P(t)=\left\{P_{l}(t)\right.$, $\left.P_{2}(t), \ldots P_{r}(t)\right\} P i(t)$ is the probability of selecting action $a \in A$ at time instant $t_{i}$.

$G=Q=A$ is the output function. $\mathrm{G}$ is deterministic one-toone function.

\section{$E=$ estimator containing environmental characteristics}

Using the learning automation, an estimator stochastically computes the output function obtainable in a bandwidth usage environment for a set of possible environment responses on the radio. With the CRN models $R_{1, \ldots, R_{n}}$, the estimator is updated. Consequently, it adapts to environmental changes such as ABR bandwidth and is implemented by the learning algorithm $\mathrm{T}$ presented in fig. 2. It is used for obtaining the estimator vector

$$
U(t)=U_{1}(t), U_{2}(t), \ldots, U r(t)
$$

Where

$U(t)$ is the estimator vector at any time instant $t$, and

$$
E(t)=D^{\prime}(t), M(t) U(t)
$$

Where

$D^{\prime}(t)$ is the deterministic estimator vector at any time $t$; $D(t)=\left\{d^{\prime},(t), d^{\prime}{ }_{2}(t), \ldots, d^{\prime} r(t)\right\}$ and

$$
\begin{gathered}
M(t)=\left\{M_{l}(t), \quad M_{2}(t), \quad M r(t)\right\} \quad \text { is oldness vector; } \\
M i(t)=t-\max \{j: j<t ; a(j)=a i\}
\end{gathered}
$$


Combining equations (1) and (2), algorithmic description of $\mathrm{T}$ is given in fig. 2 .

Initialization: all $\mathrm{Pi}=1 / \mathrm{r}$

Step 1: Select an action $a(t)=a k$

Step 2: Get feedback $b(t) \epsilon(0,1)$ from environment

Step 3: Computer new deterministic estimate dik(t)

Step 4: Update oldness vector $\operatorname{Mi}(t)=m(t-1)+1$

Step 5: For every ai $(i=1,2, \ldots, r)$, compute new estimate Ui $(t)$

Step 6: Select optimal action am with highest estimate Um = max $\{U i(t)\}$

Step 7: Update the probability vector

Fig. 2. Learning Algorithm for Automation

\section{A. Discussion on CR-based model}

The physical architecture of cognitive radio (CR) in ad-hoc setups make it feasible for receiving wideband signal. As software defined radio (SDR), with its radio frequency (RF) frontend, it is equipped with the capability to detect any weak signal in large dynamic range. This communication model can tune to any frequency band to receive any modulation.

As the estimator will be updated by the ABR connection source parameters - bandwidth resources are reserved for CBR and VBR connection that will be set up and the bandwidth becomes free again when CBR and VBR connections are released. This non-reserved bandwidth made available to ABR connections make all traffic sharable.

\section{B. Modalities for defined etiquettes}

Using BGP routing protocol, the cognitive-based service network and its special feature integrates well with other routing protocols. BGP also enables routing across all Internet service and other network providers. Combining with other technologies (WLAN, spread signal, infrared, WiMAX etc), temporarily unused band is used by any of the opportunistic radio, based on defined etiquettes to improve overall spectrum utilization [18].

As the estimator is updated by ABR connection source parameters - bandwidth resource is reserved for CBR and VBR connections set up and it becomes free again when CBR and VBR connections are released. This non-reserved bandwidth made available to ABR connections make virtually all traffic sharable.

To evaluate the 'commons' management regime, the following application specifications supported in unlicensed spectrum, under well defined protocols:

- Wi-Fi model of unlicensed devices - promotes innovation in wireless devices and IT business;

- mobile operators sharing of $3 \mathrm{G}$ spectrum minimizes transaction costs for accessing spectrum individually;

- realization of community mesh networks - provides mechansms for managing congestions, emphasizing co-ordination in co-existence.
- reliance on industry standardization process - fosters spectrum-specific etiquettes of management since the 'commons' regime also require specialized mechanisms.

\section{CONCLUSION}

The capability of cognitive radio (CR) within the wireless traffic provides many of current wireless systems with adaptability to existing spectrum allocation and overall spectrum utilization. CR supports common channels signalling; enabled with consistent security and privacy, envisioned in secured BGP [16].

Also, the commons spectrum will be more attractive to applications, which are adaptive and reasonably tolerant to congestion [19]. The system therefore, having mechanism for allocating resources among users/uses is equipped with established procedures to verify protocol is in conformance with agreed etiquettes.

With licensed wireless environment there are increasing demand and use of heterogeneous devices, uses leading to relatively insufficient spectrum. Spectral usage will be more efficient and spectral scarcity alleviated for broadcast and communication networks if suggested model is adopted. Users will benefit more significantly. Strategies to enhance wireless mobility management for qualitative seamless roaming and service continuity are suggested for future research.

\section{ACKNOWLEDGMENT}

We are grateful to the management of Rufus Giwa Polytechnic, Owo, Nigeria and Tertiary Educational Task Fund (TETFUND) Nigeria for the academic staff training and development fund made available to publish this research paper.

\section{REFERENCES}

[1] S. M. Benjamin, "Spectrum Abundance and the choice between Private and Public Control", New York University Law Review, vol 78, 2007.

[2] W. Lehr and J. Crowcroft "Managing Shared Access to a spectrum Common", Working Paper Series, ESD-WP-2007-01, Cambridge University, 2006 and 2007.

[3] C. Liu and J. Kaiser J., "A survey of mobile ad-hoc network routing protocols", University of Magdeburg, Technical representation, 2005.

[4] J. Haillot and F. Guidec F., "A protocol for content-based communication in disconnected mobile ad-hoc networks, Journal of Mobile Information System, vol 6, no2, 2010, pp 123-154.

[5] A. Benchi, P. Launay P. and F. Guidec F., "A JavaSpace Implementation for opportunistic Networks" Proceedings of the International Conference on Future Computational Technologies \& Applications, FUTURE COMPUTING, 2012, pp 49-54.

[6] P. Rysavy, "Breakfree with wireless LANs network computing, mobile and wireless technology feature", 2009, www.rysavy.com/articles/Breakfree.html.

[7] G. Faulhaber, "The Question of Spectrum: Technology, Management, and Regime Change", paper presented at Michigan University, May 16, 2005.

[8] E. Goodman, "Spectrum Rights in the Telecoms to come", San Diego Law Review vol 41, 2004, pp 269-404.

[9] E. Friedman, "Fair and Robust Power Allocation Rules for Multiple Access Channels", Draft, Operation Research \& Industrial Engineering, Cornell University, 2005.

[10] A. Odlyzko, "Telecom Dogmas and Spectrum Allocations", 2004, Wireless unleashed blog www.dtc.umn.edu/doc/network.html 
[11] D. Reed, "How Wireless Networks Scale: the Illusion of Spectrum Scarcity", 2002 , Washington, www.jacksournet/spectrumcapacity/FCC.pdf

[12] D. Reed, "Liquid Protocols", MIT Cambridge Communication , 2005, www.cfp.mit.edu/slides/David_reed_Jun5.pdf.

[13] H. Demstz, "Towards a Theory of Property Rights: competition between private and collective ownership", Journal of Legal Studies, vol XXX1(2), 2000, pp 653-672.

[14] F. Kelly, "Models for a self-managed Internet" Philosophical Transactions of the Royal Society, 2000, A358.

[15] M. Lepinski and S. Kent, "An infrastructure to support Secure Internet Routing” RFC 6480 February, 2012.
[16] J. Durand, I. Pepelnjuk and G. Doering G., "BGP Operations and Security; Internet Draft, IETF, www.ietf.org/html/draft/bgp.txt retrieved June 30, 2014.

[17] F. J. Ogwu, M. Talib M. and G. A. Aderounmu, "Stochastic Estimatorbased Wireless Traffic Control Scheme", Journal of Computer Science, 3(12), 2007, pp 918-923.

[18] K. Horiokisio, "WiMax Networks and Cognitive Radio research", 2013, www.cmpe.boun.edu/wico=research.

[19] D. P. Sataparthy, "An Algorithm for Unlicensed Fixed Power Devices", IEEE Wireless Communications and Networking Conference (WCNC), September, 2002. 